\title{
Les Taeniopterygidae du bassin du Duero (Insecta : Plecoptera)
}

\author{
C. Berthélemy ${ }^{1}$ \\ M. Gonzàlez del Tánago²
}

Les auteurs donnent la distribution de sept espèces de Taeniopterygidae dans trente-neuf sta tions situées sur le Duero et ses affluents. Ils décrivent Brachytera vera nov. sp. et discutent des caractères spécifiques de quelques autres Brachypterinae.

\section{The Taeniopterygidae of the Duero Basin (Insecta : Plecoptera).}

Seven species of Taeniopterygidae are recorded from thirty nine stations on the River Duero and its tributaries. Brachyptera vera nov. sp. is described and the specific characters of some other Brachypterinae are discussed.

Des prélèvements de faune benthique complétés par quelques captures d'adultes ont été effectués dans toute la partie espagnole du Bassin du Duero (fig. 1). Parmi les Plécoptères récoltés figurent sept espèces de Taeniopterygidae, dont une nouvelle espèce de Brachyptera que nous décrivons ci-après.

\section{Liste des stations}

\section{Station I: Rio Duero à Hinojosa de la Sierra (Soria), alti- tude $1080 \mathrm{~m}$. \\ Station 2: Rio Duero à Garray (Soria), $1010 \mathrm{~m}$. \\ Station 3: Rio Duero à Tardajos (Soria), $980 \mathrm{~m}$. \\ Station 4: Rio Duero à Andaluz (Soria), $900 \mathrm{~m}$. \\ Station 5: Rio Duero à San Esteban de Gormaz (Soria), $860 \mathrm{~m}$. \\ Station 6: Rio Duero à Roa (Burgos), $750 \mathrm{~m}$ \\ Station 7: Rio Ucero à Burgo de Osma (Soria), $890 \mathrm{~m}$. \\ Station 8: Rio Pisuerga à Cordovilla la Real (Palencia), $770 \mathrm{~m}$. \\ Station 9: Rio Pisuerga à Soto de Cerrato (Palencia),} $740 \mathrm{~m}$.

1. Laboratoire d'Hydrobiologie (E.R.A. 702 du C.N.R.S.), Université Paul Sabatier, 118, route de Narbonne, 31062 Toulouse Cedex, France.

2. Laboratorio de Zoologia y Entomologia, Escuela Tecnica Superior de Ingenieros de Montes, Universidad Politecnica de Madrid, (Ciudad Universitaria), Madrid 3, España.
Station 10: Rio Arlanza à Quintanar de la Sierra (Soria), $1090 \mathrm{~m}$.

Station 11: Rio Arlanza a Torrepadre (Burgos), $790 \mathrm{~m}$.

Station 12: Rio Arlanza à Quintana del Puente (Palencia), $770 \mathrm{~m}$.

Station 13: Rio Arlanzón à Pineda de la Sierra (Burgos), $1200 \mathrm{~m}$.

Station 14: Rio Carrión à Villoldo (Palencia), $790 \mathrm{~m}$.

Station 15: Rio Valderaduey à Sahagún (León), $810 \mathrm{~m}$.

Station 16: Rio Valderaduey à Benegiles (Zamora), $650 \mathrm{~m}$.

Station 17: Rio Esla à Riano (León), $1090 \mathrm{~m}$.

Station 18: Rio Esla à Gradefes (León), $870 \mathrm{~m}$.

Station 19: Rio Esla à Villarroane (León), $790 \mathrm{~m}$.

Station 20: Rio Esla à Ardón (León), $780 \mathrm{~m}$.

Station 21: Rio Esla à Santa Colomba de las Carabias (Zamora), $730 \mathrm{~m}$.

Station 22 : Rio Esla à Bretó (Zamora), $690 \mathrm{~m}$.

Station 23 : Rio Cea à Almanza (León), $900 \mathrm{~m}$.

Station 24: Rio Cea à Valderas (León), $720 \mathrm{~m}$

Station 25: Rio Porma à Vegas del Condado (León), $870 \mathrm{~m}$.

Station 26: Rio Orbigo à Carrizo de la Ríbera (León), $880 \mathrm{~m}$.

Station 27: Rio Orbigo à Santa Cristina de la Polvorosa (León), $740 \mathrm{~m}$.

Station 28: Rio Tera à Trefacio (Zamora), $900 \mathrm{~m}$.

Station 29: Rio Tera à Mozar (Zamora), $690 \mathrm{~m}$.

Station 30: Rio Riaza à Riofrio de Riaza (Segovia), $1150 \mathrm{~m}$.

Station 31: Rio Riaza à Berlangas de Roa (Segovia), $770 \mathrm{~m}$.

Station 32 : Rio Duratón à Duruelo (Segovia), $1000 \mathrm{~m}$.

Station 33: Rio Cega à Rebollo (Segovia), $900 \mathrm{~m}$.

Station 34: Rio Adaja à Muñotello (Avila), $1100 \mathrm{~m}$.

Station 35: Rio Adaja à Valdestillas (Valladolid), $690 \mathrm{~m}$.

Station 36: Rio Tormes à Angostura (Avila), $1180 \mathrm{~m}$.

Station 37: Rio Tormes à Ejeme (Salamanca), $850 \mathrm{~m}$.

Station 38: Rio Huebra à Tamames (Salamanca), $840 \mathrm{~m}$.

Station 39: Rio Agueda à El Payo (Salamanca), $810 \mathrm{~m}$. 


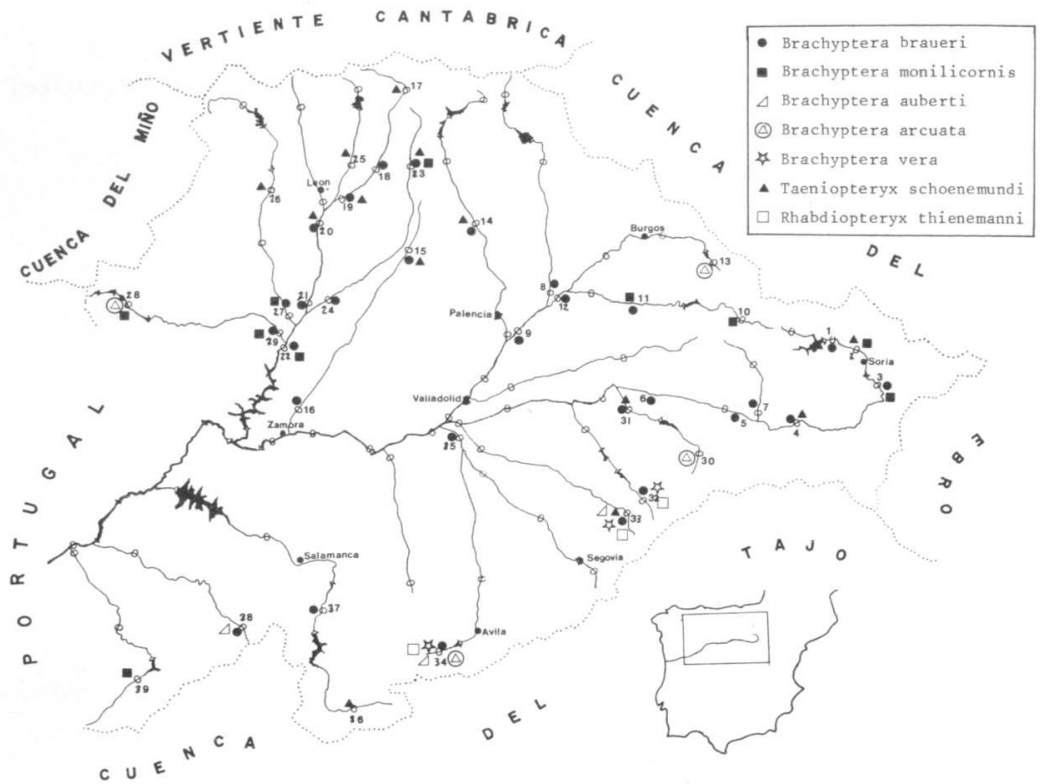

Fig, 1. Répartition des Taeniopterygidae dans le bassin du Duero.

\section{Brachyptera arcuata (Klapálek, 1902)}

L'allongement des articles antennaires et la réduction de la sclérification abdominale montrent que cet endémique ibérique appartient au même groupe que Brachyptera risi (Morton) et $B$. seticomis (Klapálek). Les antennes sont moins étroites, toutefois, que chez ces deux espèces et le canal de la spermathèque est dépourvu de microtriches (Berthélemy et Terra 1980). La souche de B. arcuata s'est donc isolée assez précocement, avant l'apparition de ces caractères dans la lignée menant à $B$. risi et $B$. seticomis.

St. $13: 23.2 .1981,1$ larve (L.). - St. $28: 20.2 .1981,3$ L. -St. $30:$ 19.3.1981, 1 L. - St. $34: 3.3 .1982,8$ L. ; 7.3.1982, 6 L. ; 25.4.1982, $1 \circ 7$ \&.

Brachyptera auberti (Consiglio, 1957)

Déjà signalée de la partie portugaise du bassin du Duero (Terra 1979), B. auberti remplace B. risi en Sar- daigne, en Afrique du Nord et dans la Péninsule Ibérique au sud du bassin de l'Ebre.

St. 33 : 3.3.1981, 4 L. ; 19.3.1981, 8 L. - St. 34 : 15.3.1981, 1 L. ; $3.3 .1782,8$ L. ; $7.3 .1982,1$ L. ; 19.3.1982, 2 o , 1 \& ; 25.4.1982, 1 \&. - St. 38: 21.3.1981, 5 L.

Brachyptera brauerí (Klapálek, 1900)

Les larves suédoises et luxembourgeoises de $B$. braueri présentent des taches à contour vague et mal défini (Brinck 1949, Hoffmann 1960, fig. 11). Les larves suisses de $B$. monilicornis, d'autre part, ont des dessins dorsaux nets (Aubert 1946, reproduit par Illies 1955, Aubert 1959 et Hoffmann 1960). Dans la Péninsule Ibérique, au contraire, les larves de $\boldsymbol{B}$. braueri (fig. 4 b) ont une pigmentation plus contrastée que celles de B. monilicomis (fig. $4 \mathrm{~m}$, voir aussi Zwick 1972). La coloration ne peut servir de critère de détermination dans une région donnée qu'après avoir été observée sur des larves mâles au dernier stade. 
St. 1 : 23.2.1981, 1 L. - St. 3: 24.2.1981, 111 L. - St. 4 : 24.2.1981, 77 L - St. $5: 25.2 .1981,141$ L. - St. $6: 25.2 .1981$, 7 L. - St. $7: 25.2 .1981,2$ L. - St. 8: 7.3.1981, 1 o, 56 L. - St. 9:7.3.1981, 30 L. - St. II:7.3.1981,6 \%. 4 Q. 153 L. - St. $12: 7.3 .1981,53$ L. - St. $14: 21.2 .1981,41$ L. St. J5 : 19.2.1981, 43 L. - St. $16: 21.2 .1981,9$ L. - St. 18 : 17.2.1981, 45 L. - St. 19: 19.2.1981, 60 L. - St. 20: 19.2.1981, 14 L. - St. $21: 20.2 .1981,7$ L. - St. $23: 19.2 .1981$, 153 L. - St. $24: 20.2 .1981,59$ L. - St. $27: 20.2 .1981,12$ L. - St. 29 : 20.2.1981, 6 L. - St. 31 : 25.2.1981, 2 L. - St. 32 : 19.3.1981, 10 L. - St. $33: 3.3 .1981,3 \sigma, 3$ \%, 4 L. ; 19.3.1981, $1 \sigma .19 \mathrm{~L}$-St. $34: 15.3 .1981,1 \sigma .47$ L. -St. $35: 6.3 .1981$, 8 L. - St. $37: 21.3 .1981,2$ L. $-S t .38: 21.3 .1981,13$ L.

\section{Brachyptera monilicornis (Pictet, 1841)}

Espèce déjà récoltée en Espagne et au Portugal, y compris dans le bassin du Duero (Zwick 1972, Terra 1979, Berthélemy et Terra 1980).

St. 2 : 24.2.1981, 148 L. - St. $3: 24.2 .1981,4$ L. - St. I0: 23.2.1981, 7 L. - St. 11: 7.3.1981, 1 \%, 6 L. - St. 23 : 19.2.1981, 53 L. - St. $27: 20.2 .1981,7$ L. - St. $28: 20.2 .1981$, 2 L. - St. $29: 20.2 .1981,16$ L. - St. $39: 21.3 .1981,5$ L.

\section{Brachyptera vera nov, sp.}

Mâles, 8,5 à $9 \mathrm{~mm}$; femelles, 9,5 à 10,5 mm.

Articles 3 à 5 du flagelle antennaire un peu plus longs que larges (rapport longueur/largeur compris entre 1,1 et 1,25 ), dilatés à l'apex mais non globuleux (fig. $2 \mathrm{~A}$ ).

Tête jaune et brune, à dessins nets. Le pigment brun se trouve dans le triangle inter-ocellaire et sur les callosités frontales, tentoriales et occipitales. L'ensemble rappelle $B$. braueri (Despax 1951, Winkler 1957, Hoffmann 1960). Pronotum éclairci sur le pourtour et au centre de chaque moitié du sclérite. Sclérites méso- et métathoraciques bruns. Pattes assombries au niveau du genou et du tarse.

Ailes longues chez les femelles ( 10 à $11 \mathrm{~mm}$ ), courtes ( 6 à $6,5 \mathrm{~mm}$ ) mais de forme normale chez les mâles ${ }^{3}$, comme chez B. putata (Newman) - voir Hynes (1957), contrairement à l'indication de Ricker et Ross (1975). Trois bandes transversales et apex enfumés. Une quatrième bande, moins nette, se trouve juste avant la tache apicale, comme chez $B$. braueri, $B$. monilicornis et B. starmachi (Sowa 1966, fig. 3). A l'aile antérieure, $R_{2}+{ }_{3}$ est ramifiée une fois sur

3. L'epithete specifique souligne l'adequation entre le nom générique et la petite taille des ailes du mâle, caractère peu fréquent chez les Brachypiero. quatre et la première cubitale comporte quatre, plus rarement cinq nervures.

Mâle.

Echancrures des tergites abdominaux moderées, moins profondes que chez $B$. risi. Prolongements du tergite 10 courts (fig. $2 \mathrm{C}$ et $\mathrm{D}$ ). Bulbe basal de l'épiprocte renflé. Tige du procès sclérifié symétrique, de largeur à peu près constante. Apex plus saillant en avant qu'en arrière (fig. $2 \mathrm{I}$ ), formant en vue dorsale un $\mathrm{U}$ épaissi en avant (fig. $2 \mathrm{~J}$ ). Canal de l'ampoule interne débouchant près de l'apex. Filaments dévaginables un peu plus courts que chez $B$. braueri (voir Berthélemy 1980, fig. 23). Cerques courts, avec un appendice basal petit, globuleux, peu sclérifié, situé au-dessus du cerque et n'en dépassant pas l'extrémité vers l'arrière.

Sternites abdominaux grands. Taches latérales du sternite $9 \mathrm{mal}$ délimitées (fig. 2 E). Prolongement sous-génital arrondi, avec un cul-de-sac peu profond et un bord postérieur échancré. Formations paraproctales rappelant celles de $B$. braueri mais avec un flagelle plus court ( $f$ ig. $3 \mathrm{v}$ ).

Femelle.

Sternites abdominaux constituant une bande transversale complète. Sclérites vulvaires assombris. De part et d'autre, deux taches latérales sont souvent prolongées en avant et en arrière pour former des bandes longitudinales (fig. $2 \mathrm{H}$ ). Bourrelets vulvaires couverts de mamelons comme chez $B$. braueri (Berthélemy 1980, fig. 11). Canal de la spermathèque un peu plus court que chez $B$. braueri, sans microtriches sur la face interne. Sternite 9 à bords latéraux concaves, à bord postérieur en angle obtus, parfois plus arrondi que sur la figure $2 \mathbf{H}$. Larve.

Pigmentation faible, peu contrastée (fig. 4 v). Tête plus foncé que le reste du corps. Articles antennaires courts, comme chez $B$. braueri et $B$. monilicornis (Berthelemy et Laur 1976, fig. $1 \mathrm{~m}$ ). Franges natatoires des tarses plus clairsemées que chez ces deux espèces. Soies dressées des tergites abdominaux courtes et arrondies (fig. $4 \mathrm{~s}$ ). Pas de longues soies dorsales à la base des cerques. Prolongement du neuvième sternite abdominal anguleux chez la larve femelle, plus arrondi chez la larve mâle. Crochets des paraproctes de cette dernière en demi-cercle, un peu dissymétriques et disposés dans un plan presque frontal. Premier article des cerques de la larve mâle à peine dilaté. 


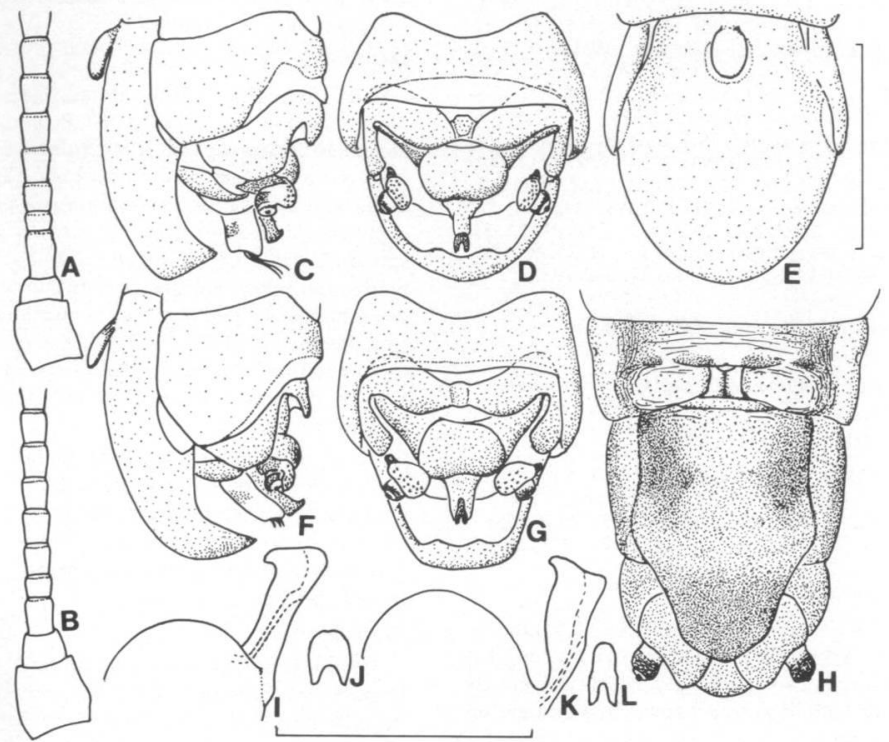

Fig. 2. Brachyptera et Rhabdiopteryx. A : partie basale de l'antenne d'un mâle de Brachyptera vera. - B : id., Rhabdiopteryx neglecta. - C : extrémité postérieure de l'abdomen d'un mâle de $B$. vera, profil gauche, formations paraproctales non figurées. - D : id., face dorsale. - E : $i d$., face ventrale. - F : id., B. olgirica, profil gauche. - G : id., face dorsale. - H ; id., B. vera femelle, face ventrale. - I : procès sclérifié de l'épiprocte d'un mâle de $B$. vera, profil gauche; en tirets, canal de l'ampoule interne. - J : id., apex. - K : id., B. algirica, profil gauche. - L : id. apex. Le trait vertical (fig. A à $\mathrm{H}$ ) correspond à $1 \mathrm{~mm}$, le trait horizontal $(f i g . \mathrm{I}$ à $\mathrm{L}$ ) à $0,5 \mathrm{~mm}$.

La larve de $B$. vera ressemble à celle de $B$. starmachi mais le prolongement du sternite 9 de $B$. vera est plus étroit, chez les deux sexes. En outre, $B$. starmachi n'a jamais été trouvée à l'ouest des Carpathes et les aires de répartition des deux espèces ne se chevauchent certainement pas.

La larve de $B$. vera se distingue de celles des autres Brachyptera ibériques par l'absence de longues soies dorsales à la base des cerques. Elle diffère de la larve de Rhabdiopteryx thienemanni par sa coloration plus claire, l'angle plus ouvert de la suture postfrontale, le nombre plus élevé des courtes soies dressées de la surface des tergites abdominaux et la forme dilatée de ces soies.

\section{Discussion.}

Des caractères synapomorphes permettent de définir deux lignées dans le genre Brachyptera. Les articles antennaires sont allongés et la face ventrale de l'abdomen est peu sclérifiée chez $B$. arcuata, $B$. auberti, B. beali (Navás), B. galeata Koponen, $B$. graeca Berthélemy, $B$. helenica Aubert, $B$. phthiotica Berthélemy, $B$. risi, $B$. seticomis et quelques autres espèces que nous n'avons pas eu l'occasion d'exa. miner. Les articles antennaires sont devenus globuleux chez $B$. braueri, $B$. monilicornis, $B$. putata, $B$. starmachi et $B$. trifasciata (Pictet). Les formations paraproctales de $B$. starmachi (fig. 3 s) sont intermédiaires entre celles de $B$. braueri et de $B$. monili- 
Fig. 3. Vues dorso et ventropostérieures des formations paraproctales de mâles de Brachyptera vera (v), B. algirica (a), B. starmachi (s), Rhabdiopteryx thienemanni (t) et $R h$. neglecta (n). Le trait correspond à $0,5 \mathrm{~mm}$.

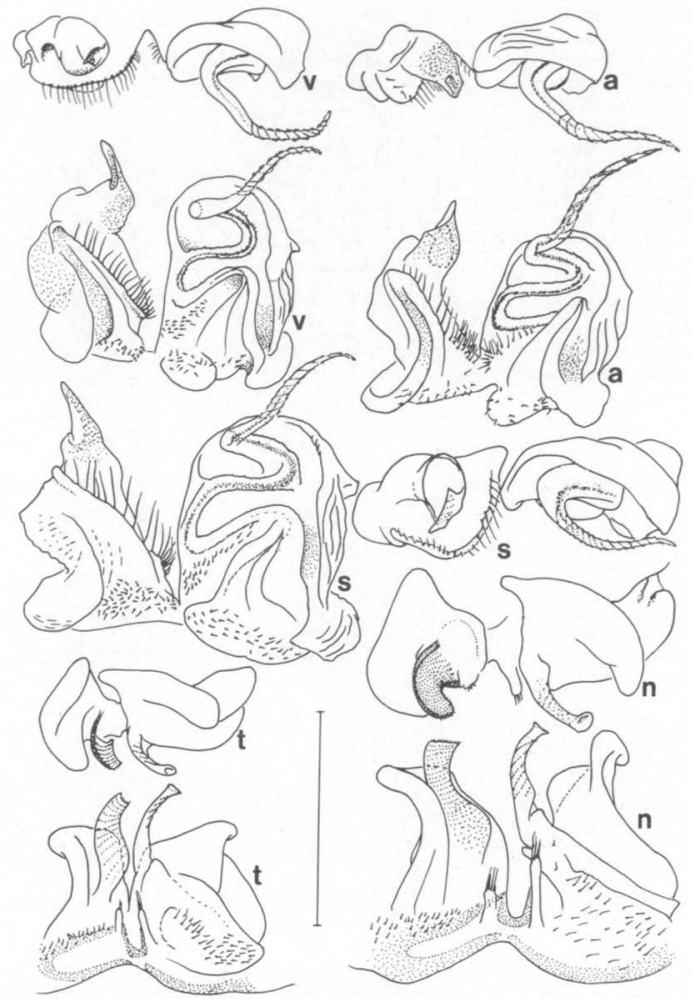

cornis (Berthélemy 1980) et confirment les affinités de ces trois Brachyptera.

Les relations phylogéniques de quelques espèces ne peuvent être définies à l'aide des caractères précédents. Les articles antennaires et les sternites abdominaux ont en effet conservé une structure primitive, proche de celle des Rhabdiopteryx (fig. 2 B), chez B. algirica, Aubert, B. tristis (Klapálek) - voir Kis 1974, fig. $20 \mathrm{~A}$ - et chez B. vera (fig. 2 A).

$B$. vera se distingue des trois autres espèces précitées par la coloration contrastée de la tête, la réduction des ailes du mâle, la forme du procès sclérifié de l'épiprocte et la petite taille des appendices basaux des cerques.

Le mâle de $B$. algirica a été décrit d'après un « pharate adult * et Aubert (1956) n'a représenté que l'apex du procès sclérifié de l'épiprocte. Nous donnons ici des figures complémentaires, d'après deux mâles du Haut Atlas marocain (fig. 2 F, G, K et $\mathbf{L}$ ). Le bord postérieur de la plaque sous-génitale de $B$. algirica est plus échancré et ses angles latéraux sont plus aigus que chez $B$, vera. 
Fig. 4. Larves au dernier stade de Brachyptera.

En haut, pigmentation dor. sale de larves mâles de $B$. vera $(v), B$. braueri (b) et $B$. monilicornis $(\mathrm{m})$. Le trait correspond a $5 \mathrm{~mm}$. En bas. extrémités postérieures de l'abdomen de larves de $B$. vera. De gauche à droite, face ventrale de la larve femelle, face ventrale et profil gauche de la larve mâle (le trait correspond à $1 \mathrm{~mm}$ ) ; soies (s) du bord du tergite 10, près de la ligne médiane (le trait correspond a $\mathbf{5 0}$ $\mu \mathrm{m})$.

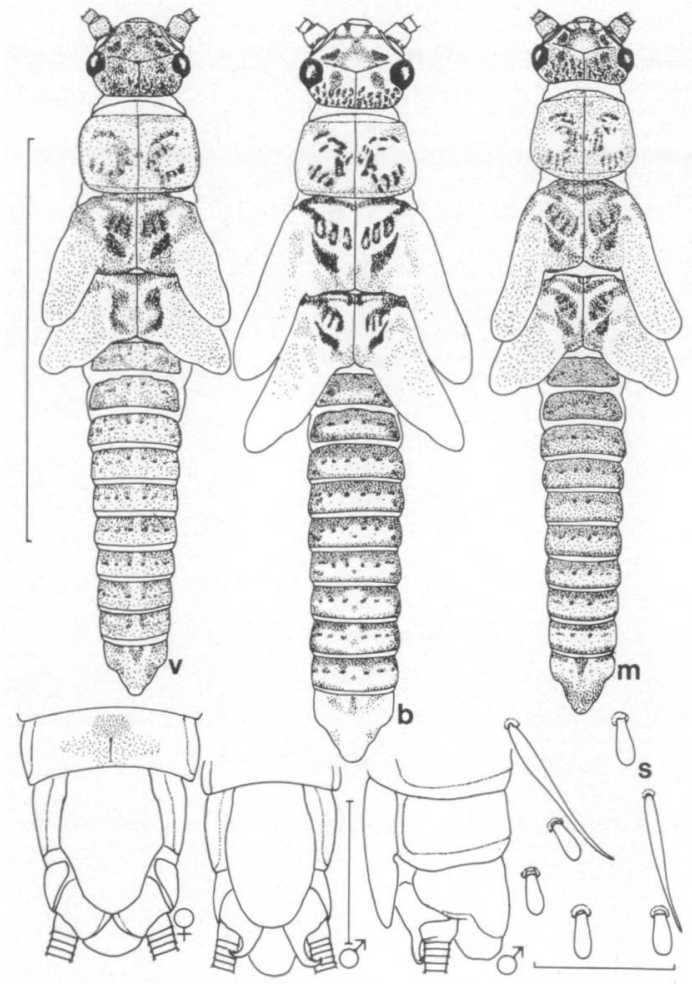

Chez $\boldsymbol{B}$. dinarica, d'autre part, les prolongements du tergite 10 et les filaments sclérifiés dévaginables de l'épiprocte sont plus longs, la tigelle de l'épiprocte est plus épaisse et le cul-de-sac de la plaque sous-génitale est plus profond que chez $B$. vera. Les différences entre la femelle de $B$. vera et celle de $B$. dinarica devront être précisées ultérieurement. Les femelles attribuées à $B$. dinarica par Aubert (1964) n'ont pas été capturées dans la même localité que le mâle et appartiennent peut-être à $B$. tristis plutôt qu'à $B$. dinarica.
La forme des soies des larves, la tache apicale des ailes et l'alignement des soies sur la formation paraproctale gauche des mâles semblent être des caractères synapomorphes qui rattachent $B$. vera au groupe braueri-starmachi-monilicornis.

$B$. vera a été trouvée vers $1000 \mathrm{~m}$ d'altitude dans trois ruisseaux temporaires du versant nord de la cordillère transversale qui sépare le bassin du Duero de celui du Tage (fig. 1) ${ }^{4}$.

4. Une race de B. vera chez laquelle le mále est macroptère vient d'être découverte dans la province de Cordoue. 
Holotype : un mále récolté le 19.3.1982 près du Rio Adaja à Muñotello (St. 34). Paratypes : 20 et 4 \& de la même récolte ; 1 \& capturée le 15.3.1981 dans la même station; 1 or (19.3.1981) et 1 \& (3.3.1981) de la station 33 .

Autre matériel : St. $32: 3.3 .1981,1$ L. ; 3.3.1982, 6 L. St. $33: 3.3 .1981,7$ L. ; $19.3 .1981,1 \mathrm{~L}$.

Rhabdiopteryx thienemanni Illies, 1957

Les formations paraproctales des mâles (fig. $3 \mathrm{t}$ ) sont proches de celles de $R h$. neglecta (fig. $3 \mathrm{n}$ et Zwick 1973, fig. 50 F). Les pavillons de $R h$. thienemanni sont plus épais et moins enveloppants. Le pavillon droit présente un repli externe supplémentaire.

Les critères proposés pour distinguer les larves des Rhabdiopteryx de celles des Brachyptera ne s'appliquent que dans des régions restreintes (Tyrol, Kühtreiber 1934 ; Allemagne, Illies 1955 ; Grande Bretagne, Hynes 1957 ; Suisse, Aubert 1959). En effet, la suture post-frontale est anguleuse chez $B$. trifasciata comme chez les Rhabdiopteryx (Aubert 1959). La face ventrale est claire chez $R h$. acuminata (Hynes 1957, sub nom. Rh. anglica) et chez Rh. thienemanni, comme chez les Brachyptera. Les longues soies dorsales de la base des cerques manquent chez B. starmachi et $B$. vera, comme chez les Rhabdiopteryx. Enfin, contrairement à ce qu'indique Hynes (1957) pour $R h$. acuminata, $R$ h. thienemanni possède quelques courtes soies dressées sur les tergites abdominaux. Ces soies sont moins nombreuses, toutefois, que chez les Brachyptera. A l'échelle européenne, il faut donc considérer globalement les caractères spécifiques des larves de Brachypterinae sans que les genres puissent être déterminés au préalable.

St. $32: 12.2 .1981,1 \sigma, 7$ Q $5 \mathrm{~L}$ : $3.3 .1981,3 \sigma, 3 \%, 18 \mathrm{~L}$. 19.3.1981, $2 \sigma, 2$ ๑, 17 L. - St. $33: 3.3 .1981,4$ L. ; 19.3.1982, $1 \propto, 4$ ९. - St. $34: 15.3 .1981,22$ L. ; 25.4.1982, $1 \%$.

Taeniopteryx schoenemundi (Mertens, 1923)

Cette espèce, dont la présence dans la Péninsule Ibérique n'a été signalée qu'assez récemment (Berthélemy et Terra 1980), est le Taeniopterygidae le plus répandu dans le bassin du Duero après Brachyptera braueri.

St. 2 : 24.2.1981, 5 L. -.St. 4 : 24.2.1981, 1 L. - St. $14:$ 21.2.1981, 4 L. - St. 15: 19.2.1981, 2 L. - St. $17: 17.2 .1981$, 1 L. - St. 19: 19.2.1981, 2 L. - St. $20: 19.2 .1981$, 1 L. St. $23: 19.2 .1981,8$ L. - St. $25: 1.2 .1981,6$ L. - St. 26 : 18.2.1981, 3 L. - St. $31: 25.2 .1981,37$ L. - St. $33: 19.3 .1981$, $6 \circ, 2$ \&, 2 L.; 19.3.1982, $1 \propto$ \%, 2 \&. $-S t .36: 15.3 .1981$, 1 \&, $1 \mathrm{~L}$.

\section{Conclusion}

La faune la plus diversifiée se rencontre dans la partie amont des cours d'eat, en particulier dans les ruisseaux temporaires du flanc sud du bassin (stations 32, 33 et 34, voir fig. 1). L'arrêt de l'écoulement en été élimine probablement certains concurrents et surtout certains prédateurs potentiels des Brachypterinae. La faune s'appauvrit en aval, où Brachyptera braueri reste seule fréquente. C'est une espèce fluviatile dans le reste de l'Europe également (Berthélemy 1966). B. monilicornis cohabite ça et là avec $B$. braueri, sans que ses exigences écologiques propres n'apparaissent clairement.

Au point de vue faunistique, la découverte de $B$. vera porte à huit le nombre des Brachyptera ibériques. Cette diversité est voisine de celle de la Péninsule Balkanique et supérieure à celle de l'Europe moyenne. Ce type de distribution est rare chez les Plécoptères et peut être lié au mode de nutrition des Brachyptera. Les larves de ce genre, en effet, ne se nourrissent pas de feuilles mortes comme la plupart des autres Nemouroidea mais elles raclent les algues qui se développent abondamment dans les cours d'eau peu ombragés des régions méditerranéennes. Pour exploiter ce milieu, une phase du cycle, presque certainement l'œuf, doit résister au ralentissement du courant et aux fortes températures estivales. Ce dernier point devra faire l'objet d'études ultérieures sur les espèces du sud de l'Europe.

\section{Remerciements}

Nous sommes très reconnaissants aux Drs J. Giudicelli (Marseille), A. Kaltenbach (Naturhistoriches Museum Wien), R. Sowa (Krakcw) et P. Zwick (Schlitz) d'avoir bien voulu nous communiquer des exemplaires de Brachyptera alginica, B. dinarica et $B$. stamachi.

Travaux cliés

Aubert (J.). 1946. - Les Plécoptères de la Suisse romande. Mitt. schweiz ent. Ges., $20: 7-128+1$ carte.

Aubert (J.). 1956. - Contribution à l'étude des Plécoptères d'Afrique du Nord. Mitt. schweiz. eni. Ges., 29 : 419-436.

Aubert (J.). 1959. - Plecoptera. Insecta Helvetica, Fauna, 1 : 140 p., Lausanne.

Aubert (J.). 1963. - Les Plécoptères de la péninsule ibérique. Eos, Rev, esp. Ent., 39 : 23-107.

Aubert (J.). 1964. - Quelques Plécoptères du Muséum d'Histoire naturelle de Vienne. Annlm naturh. Mus. Wien, $67: 287-301$. 
Berthélemy (C.). 1966. - Recherches écologiques et biogéographi. ques sur les Plécoptères et Coléoptères d'eau courante (Hydraena et Elminthidae) des Pyrénées. Annls Limnol, 2 : $227-458$.

Berthélemy (C.). 1980. - Accouplement, période d'incubation et premiers stades larvaires de Brachyptera braueri et de Perlodes microcephalus (Plecoptera). Annls Limnol, 15 (1979) : 317. 335.

Berthélemy (C.) et Laur (C.). 1976. - Plecoptères et Coléoptères aquatiques du Lot (Massif Central français). Annls Limnol., 11 (1975): 263.285.

Berthélemy (C.) et Terra (L.S. Whytton da). 1980. - Plécoptères du Porugal (Insecta). Annls Limnol, 16:159-182.

Brinck (P.). 1949. - Studies on Swedish Stoneflies (Plecoptera). Opusc. ent., Suppl. $11: 1.250$.

Despax (R.). 1951. - Plécoptères. Faume de France, 55 : 280 p. P. Lechevalier, Paris.

Hoffmann (J.). 1960. - Faune des Plécopteres du Grand-Duché de Luxembourg. Archs Inst. Gr.duc. Luxembourg, $27: 121-208$.

Hynes (H.B.N.). 1957. - The British Taeniopterygidae (Plecoptera). Trans, r. ent. Soc. London, $109: \mathbf{2 3 3 . 2 4 3 .}$
Illies (J.). 1955. - Steinfliegen oder Plecoptera. Die Tienwelt Deutschlands und der angrenzenden Meeresteile, 43 : $150 \mathrm{p}$. G. Fischer, Jena.

Kis (B.). 1974. - Plecoptera. Faune Republicii Socialiste România. Insecta, 8 (7): 273 p. Bucarest.

Kühtreiber (J.). 1934. - Die Plecopterenfauna Nordtirols. Ber. naturw.med. Ver. Innsbruck, $43 / 44: 1 . \mathrm{vII}+1.219+6 \mathrm{pl}$. + 1 carte.

Ricker (W.E.) et Ross (H.H.). 1975. - Synopsis of the Brachypteri. nae (Insecta : Plecoptera : Taeniopterygidae). Canad. J. Zool. 53:132-153.

Sowa (R.). 1966. - Brachyptera starmachi sp. n., Plecoptère nou. vellement trouvé des Carpathes. Bull Acad pol. Sci. II Ser. Sci. biol. 14: 427-432.

Terra (L.S. Whytton da). 1979. - Note on the Portuguese Plecoptera. Gewäss. Abwäss., 64 : $60-68$.

Winkler (O.). 1957. - Plecoptera slovenska (Faunisticko-systematická studia). Biol. Práce, 3; 1-98.

Zwick (P.). 1972. - Plecoptera (Ins.) aus dem Mittelmeergebiet, vor allem aus Portugal und Spanien. Ciênc. biol., $1: 7-17$.

Zwick (P,). 1973. - Insecta : Plecoptera. Phylogenetisches System und Katalog. Das Tierreich, 94 : xxxii +465 p. W. de Gruyter, Berlin. 